$\begin{array}{ll}\text { Research Square } & \begin{array}{l}\text { Preprints are preliminary reports that have not undergone peer review. } \\ \text { They should not be considered conclusive, used to inform clinical practice, } \\ \text { or referenced by the media as validated information. }\end{array}\end{array}$

\title{
Combining Cell Therapy with Autologous Schwann Cell and Bone Marrow- derived Mesenchymal Stem Cell in Patients with Subacute Complete Spinal Cord Injury: Safety Considerations and Possible Outcomes
}

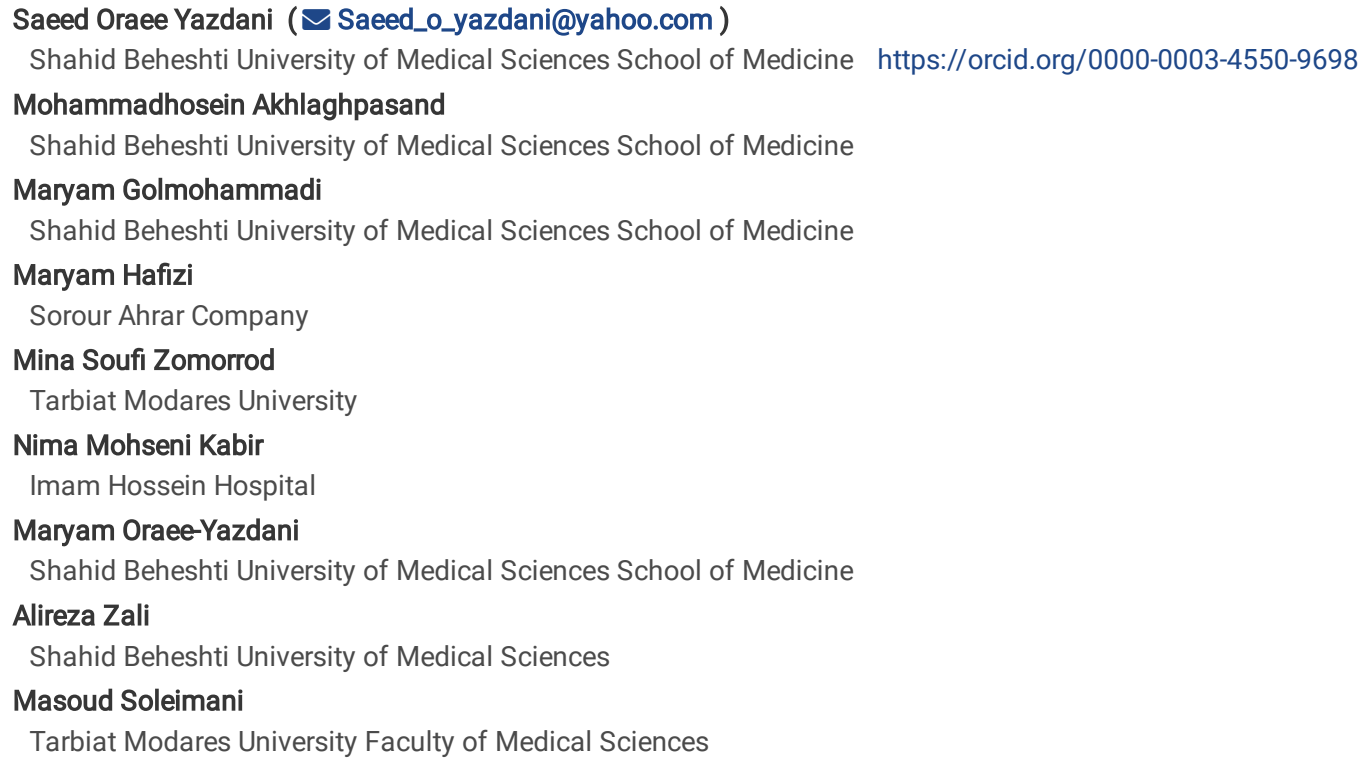

Version of Record: A version of this preprint was published at Stem Cell Research \& Therapy on August 9th, 2021. See the published version at https://doi.org/10.1186/s13287-021-02515-2. 


\section{Abstract}

Background Cellular transplantations have promising effects on treating spinal cord injury (SCl) patients. Safety alongside the complementary characteristics of Mesenchymal stem cells (MSC) and Schwann cells (SC) are suggested to be the two of the best candidates in SCI treatment. In this study, we assessed the safety and efficacy of intrathecal co-transplantation of autologous bone marrow MSC and SC in the patients with subacute traumatic complete SCI.

Methods Eleven patients with complete SCI (American Spinal Injury Association Impairment Scale (AIS); grade A) were enrolled in this study during the subacute period of injury. The patients received intrathecal autologous combination of MSC and SC and were followed-up for 12 months. We assessed neurological changes by American Spinal Injury Association's (ASIA) sensory-motor scale, functional recovery by spinal cord independence measure (SCIM-III), and subjective changes along with adverse events (AE) with our checklist. Furthermore, electromyography (EMG), nerve conduction velocity (NCV), magnetic resonance imaging (MRI), and urodynamic study (UDS) were conducted for all the patients at the baseline, 6 months, and one year after the intervention.

Results Light touch ASIA score alterations were approximately the same as the pinprick changes ( $11.6 \pm 13.1$ and $12 \pm 13$, respectively) in $50 \%$ of the cervical and $63 \%$ of the lumbar-thoracic patients and both were more than the motor score alterations $(9.5 \pm 3.3$ in $75 \%$ of the cervical and $14 \%$ of the lumbar-thoracic patients). SCIM III total scores (21.2 \pm 13.3 ) and all its sub-scores ("respiration and sphincter management" (15 \pm 9.9$)$, "mobility" (9.5 \pm 13.3$)$, and "self-care" (6 $\pm 1.4)$ ) had significant changes after cell injection. Our findings support that the most remarkable positive, subjective improvements were in trunk movement, equilibrium in standing/sitting position, sensation of the bladder and rectal filling, and ability of voluntary voiding. Our safety evaluation revealed no systemic complications and radiological images showed no neoplastic overgrowth, syringomyelia, or pseudo-meningocele.

Conclusion The present study showed that autologous SC and bone marrow-derived MSC transplantation at the subacute stage of SCI could reveal significant improvement in sensory and neurological functions among the patients. It appears that using this combination of cells is safe and effective for clinical application to the spinal cord regeneration during the subacute period.

\section{Introduction}

Spinal cord injury (SCl) is a devastating condition that leads to physical, social, and vocational impairment due to the irreversible loss of neural function below the injury site (1). Based on Lancet Neurology, the global burden of diseases (GBD), injuries, and risk factors between 1990 to 2016 , no significant change in the age-standardized incidence or prevalence of SCl was noted. However, with global population growth, the absolute number of people living with the effects of $\mathrm{SCl}$ is expected to increase (2). Most of the SCl patients suffer from profound disability and its related complications, which impacts the quality of life (3). Therefore, the functional improvement after SCl remains an important issue in recent decades. Regarding lack of capacity for central nervous system regeneration, there is no definitive cure for these disorders. Advanced therapies like cell transplantation could be a promising option for treating SCl patients(4).

Numerous studies on animal models of SCl and human patients have demonstrated that cellular transplantations for SCl treatment might provide a source of neural cells and have neuroprotective and immunomodulatory effects after injury $(5,6)$. Various cell types can be used due to their capacity for self-renewal and differentiation ability; but among them, mesenchymal stem cells (MSC) and Schwann cells (SC) have better safety alongside their complementary characteristics, so these cells are suggested to be one of the best candidates for transplantation in SCl subjects $(7,8)$.

Bone marrow MSC as a multipotent stromal cell has a potential effect to differentiate into osteoblast, adipocytes, chondrocytes, mature neurons, and glial cells (9). Many studies have shown that MSCs could be considered for the SCI treatment. Moreover, MSCs can produce various types of growth factors and neuroprotective cytokines which enable them to improve or restore damaged spinal cord function (10-12). In spite of the beneficial effects of MSC transplantation, according to the previous findings, their remyelinating ability is inadequate in $\mathrm{SCl}$ patients. The importance of remyelination in spinal cord repair after injury suggests that stem cells could be combined with remyelinating cells to improve the effectiveness of transplantation (13). SCs which are normally located in peripheral nerves could migrate and colonize in lesion sites to myelinate injured axons and are one of the suitable choices for transplantation in combination with MSCs (14-16).

Successful functional recovery in the patients suffering from SCl will most likely rely on effective treatment in the period corresponding with the natural history of neuro recovery. The clinical trials have been conducted to assess the possible outcome of combinational cell therapy for treating patients with chronic SCl. Like our previous study, they have indicated an insufficient recovery in the patients with chronic disease $(13,17)$. So in this study, we aimed to assess the safety and possible efficacy of co-transplantation of autologous bone-marrow MSC and SC in the patients with subacute traumatic complete SCI (within 12 months post-injury) (18).

\section{Patients And Methods}

\section{Study design and selection criteria}

This study was designed on the basis of Declaration of Helsinki and approved by Ethics in Medical Research Committee, Shahid Beheshti University of Medical Sciences (code of ethics: 106, registered October, 2018, ethics.research.ac.ir). All the interventions were performed after obtaining informed consent from patients.

Our inclusion criteria for the study were: 1 ) Complete SCI (ASIA A); 2 ) $\geq 3$ and $\leq 12$ months post-injury; 3 ) No improvement in sensory and motor scale after 3 months in spite of regular rehabilitation program; 3) Absence of brain disease or psychological disorders; 4) No stenosis, tethering, syringomyelia, or compression in the magnetic resonance images (MRI) of the spinal cord taken at the beginning of the study; 5 ) Absence of joint stiffness or pain, rashes, or any manifestation of rheumatologic disorders; and 6) Aged between 18-60 years old.

Page 2/13 
Study exclusion criteria were 1) Presence of any movement disorder not related to SCl; 2) A major complication such as urinary tract infection with sepsis, pneumonia, venous thromboembolism (deep vein thrombosis and pulmonary embolism), etc.; 3) Fracture of upper or lower limbs leading to deformity and ankylosis; 4) Abnormal findings on baseline complete blood count.

Patients were selected from among those with spinal cord injury who referred to neurosurgery clinic of Shohada Tajrish Hospital. Eleven patients (9 men and 2 women) with the mean age of $29.09 \pm 9.41$ years old met our inclusion and exclusion criteria and successfully enrolled in this study. Four cases of the patients had cervical and seven had thoracic lesions due to road traffic accidents and fall from the height (Table 1). 
Table 1

Demographic, ASIA, and SCIMIII results of the patients.

\begin{tabular}{|c|c|c|c|c|c|c|c|c|c|c|c|c|c|c|c|}
\hline \multirow{3}{*}{\multicolumn{2}{|c|}{$\begin{array}{l}\text { Patient } \\
\text { number }\end{array}$}} & \multirow[t]{3}{*}{ Sex } & \multirow{3}{*}{$\begin{array}{l}\text { Age } \\
\text { (year) }\end{array}$} & \multirow{3}{*}{$\begin{array}{l}\text { Cause } \\
\text { of injury }\end{array}$} & \multirow[t]{3}{*}{ Vertebra } & \multirow{3}{*}{$\begin{array}{l}\text { Interval } \\
\text { between } \\
\text { injection } \\
\text { and } \\
\text { trauma } \\
\text { (month) }\end{array}$} & \multicolumn{4}{|c|}{ Sensory status (ASIA Score) } & \multicolumn{4}{|c|}{ Motor status (ASIA Score) } & ? \\
\hline & & & & & & & \multicolumn{2}{|c|}{ Light touch } & \multicolumn{2}{|l|}{ Pin prick } & \multicolumn{2}{|c|}{$\begin{array}{l}\text { Upper } \\
\text { extremities }\end{array}$} & \multicolumn{2}{|c|}{$\begin{array}{l}\text { Lower } \\
\text { extremities }\end{array}$} & \\
\hline & & & & & & & Right & Left & Right & Left & Right & Left & Right & Left & \\
\hline \multirow[t]{3}{*}{1} & Before & Male & 18 & Accident & $\mathrm{C} 4$ & 3 & $\mathrm{~T} 4(19)$ & $\mathrm{T} 4(19)$ & $\mathrm{T} 4(19)$ & $\mathrm{T} 4(19)$ & C7(6) & C7(6) & - & - & \\
\hline & $\begin{array}{l}\text { After } \\
6 \mathrm{~m}\end{array}$ & & & & & & $\mathrm{~T} 4(22)$ & $\mathrm{T} 4(22)$ & $\mathrm{T} 4(22)$ & $\mathrm{T} 4(22)$ & C7(11) & $\mathrm{C} 7(11)$ & - & - & 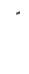 \\
\hline & $\begin{array}{l}\text { After } \\
12 \mathrm{~m}\end{array}$ & & & & & & $\mathrm{~T} 4(22)$ & $\mathrm{T} 4(22)$ & $\mathrm{T} 4(22)$ & $\mathrm{T} 4(22)$ & C8(13) & $\mathrm{C} 8(13)$ & - & - & \\
\hline \multirow[t]{3}{*}{2} & Before & Female & 42 & Accident & T3-T7 & 7 & T3(20) & $\mathrm{T} 3(20)$ & $\mathrm{T} 3(20)$ & $\mathrm{T} 3(20)$ & $\mathrm{T} 1(25)$ & $\mathrm{T} 1(25)$ & - & - & $\vdots$ \\
\hline & $\begin{array}{l}\text { After } \\
6 \mathrm{~m}\end{array}$ & & & & & & $\mathrm{~T} 4(22)$ & $\mathrm{T} 4(22)$ & $\mathrm{T} 4(22)$ & $\mathrm{T} 4(22)$ & $\mathrm{T} 1(25)$ & $\mathrm{T} 1(25)$ & - & - & $\vdots$ \\
\hline & $\begin{array}{l}\text { After } \\
12 \mathrm{~m}\end{array}$ & & & & & & $\mathrm{~T} 4(22)$ & $\mathrm{T} 4(22)$ & $\mathrm{T} 4(22)$ & $\mathrm{T} 4(22)$ & $\mathrm{T} 1(25)$ & $\mathrm{T} 1(25)$ & - & - & 4 \\
\hline \multirow[t]{3}{*}{3} & Before & Female & 38 & Accident & T10- & 3.5 & T10(34) & T10(34) & L1(40) & L1(40) & $\mathrm{T} 1(25)$ & $\mathrm{T} 1(25)$ & L1(1) & L1(1) & $\vdots$ \\
\hline & $\begin{array}{l}\text { After } \\
6 \mathrm{~m}\end{array}$ & & & & & & T10(34) & T10(34) & $\mathrm{L} 1(40)$ & L1(40) & $\mathrm{T} 1(25)$ & $\mathrm{T} 1(25)$ & $\mathrm{L} 1(1)$ & $\mathrm{L} 1(1)$ & ! \\
\hline & $\begin{array}{l}\text { After } \\
12 \mathrm{~m}\end{array}$ & & & & & & T10(34) & T10(34) & $\mathrm{L} 1(40)$ & L1(40) & $\mathrm{T} 1(25)$ & $\mathrm{T} 1(25)$ & $\mathrm{L} 1(1)$ & $\mathrm{L} 1(1)$ & t \\
\hline \multirow[t]{3}{*}{4} & Before & Male & 27 & Accident & C5-C7 & 5 & C5(8) & C6(10) & C5(8) & C5(8) & C6(5) & C6(6) & - & - & ' \\
\hline & $\begin{array}{l}\text { After } \\
6 \mathrm{~m}\end{array}$ & & & & & & C5(8) & C6(10) & C5(8) & C5(8) & C7(10) & C7(10) & - & - & 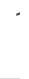 \\
\hline & $\begin{array}{l}\text { After } \\
12 \mathrm{~m}\end{array}$ & & & & & & C5(8) & C6(10) & C5(8) & C5(8) & C7(10) & C7(10) & - & - & 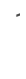 \\
\hline \multirow[t]{3}{*}{5} & Before & Male & 21 & Accident & $\mathrm{T} 12, \mathrm{~L} 1$ & 9 & L4(46) & L5(46) & L4(46) & L5(46) & $\mathrm{T} 1(25)$ & $\mathrm{T} 1(25)$ & - & - & 4 \\
\hline & $\begin{array}{l}\text { After } \\
6 \mathrm{~m}\end{array}$ & & & & & & L4(46) & L5(46) & L4(46) & L5(46) & $\mathrm{T} 1(25)$ & $\mathrm{T} 1(25)$ & L3(4) & L3(3) & ! \\
\hline & $\begin{array}{l}\text { After } \\
12 \mathrm{~m}\end{array}$ & & & & & & L4(46) & L5(46) & L4(46) & L5(46) & $\mathrm{T} 1(25)$ & $\mathrm{T} 1(25)$ & L3(5) & L3(4) & t \\
\hline \multirow[t]{3}{*}{6} & Before & Male & 30 & Accident & T6,T7 & 5.5 & T8(30) & T8(30) & T8(29) & T8(29) & $\mathrm{T} 1(25)$ & $\mathrm{T} 1(25)$ & - & - & 4 \\
\hline & $\begin{array}{l}\text { After } \\
6 \mathrm{~m}\end{array}$ & & & & & & T10(34) & T10(34) & T10(34) & T10(34) & $\mathrm{T} 1(25)$ & $\mathrm{T} 1(25)$ & - & - & 4 \\
\hline & $\begin{array}{l}\text { After } \\
12 \mathrm{~m}\end{array}$ & & & & & & T10(34) & T10(34) & T10(34) & T10(34) & $\mathrm{T} 1(25)$ & $\mathrm{T} 1(25)$ & - & - & 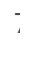 \\
\hline \multirow[t]{3}{*}{7} & Before & Male & 17 & Falling & T5,T6 & 3 & T5(24) & T5(24) & T5(24) & T5(24) & $\mathrm{T} 1(25)$ & $\mathrm{T} 1(25)$ & - & - & $\vdots$ \\
\hline & $\begin{array}{l}\text { After } \\
6 \mathrm{~m}\end{array}$ & & & & & & T9(29) & T9(29) & T9(29) & T9(29) & $\mathrm{T} 1(25)$ & $\mathrm{T} 1(25)$ & - & - & 4 \\
\hline & $\begin{array}{l}\text { After } \\
12 \mathrm{~m}\end{array}$ & & & & & & T9(29) & T9(29) & T9(29) & T9(29) & $\mathrm{T} 1(25)$ & $\mathrm{T} 1(25)$ & - & - & 4 \\
\hline \multirow[t]{3}{*}{8} & Before & Male & 30 & Accident & C5,C6 & 4 & $\mathrm{~T} 1(16)$ & $\mathrm{T} 1(16)$ & $\mathrm{T} 2(17)$ & $\mathrm{T} 2(17)$ & C7(12) & C7(12) & - & - & : \\
\hline & $\begin{array}{l}\text { After } \\
6 \mathrm{~m}\end{array}$ & & & & & & T2(18) & T2(18) & T3(19) & T3(19) & C7(12) & C7(12) & - & - & $\vdots$ \\
\hline & $\begin{array}{l}\text { After } \\
12 \mathrm{~m}\end{array}$ & & & & & & T2(18) & $\mathrm{T} 2(18)$ & T3(19) & T3(19) & C7(12) & C7(12) & - & - & $\vdots$ \\
\hline \multirow[t]{3}{*}{9} & Before & Male & 19 & Accident & $\mathrm{T} 2, \mathrm{~T} 3$ & 7 & T3(20) & T3(20) & T3(20) & T3(20) & $\mathrm{T} 1(25)$ & $\mathrm{T} 1(25)$ & - & - & $\vdots$ \\
\hline & $\begin{array}{l}\text { After } \\
6 \mathrm{~m}\end{array}$ & & & & & & T3(20) & T3(20) & T3(20) & T3(20) & $\mathrm{T} 1(25)$ & $\mathrm{T} 1(25)$ & - & - & 4 \\
\hline & $\begin{array}{l}\text { After } \\
12 \mathrm{~m}\end{array}$ & & & & & & T12(38) & L1(40) & T12(38) & L1(40) & $\mathrm{T} 1(25)$ & $\mathrm{T} 1(25)$ & - & - & 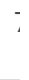 \\
\hline \multirow[t]{2}{*}{10} & Before & Male & 40 & Accident & $\mathrm{T} 11$ & 3 & T12(38) & T12(38) & T12(38) & T12(38) & $\mathrm{T} 1(25)$ & $\mathrm{T} 1(25)$ & - & - & $\vdots$ \\
\hline & $\begin{array}{l}\text { After } \\
6 \mathrm{~m}\end{array}$ & & & & & & T12(38) & T12(38) & T12(38) & T12(38) & $\mathrm{T} 1(25)$ & $\mathrm{T} 1(25)$ & - & - & ! \\
\hline
\end{tabular}




\begin{tabular}{|c|c|c|c|c|c|c|c|c|c|c|c|c|c|c|}
\hline \multirow[t]{3}{*}{11} & Before & Male & 38 & Accident & $\mathrm{C} 5, \mathrm{C} 6$ & 8 & C4(6) & C4(6) & C4(6) & C4(6) & C7(8) & C7(8) & - & - \\
\hline & $\begin{array}{l}\text { After } \\
6 \mathrm{~m}\end{array}$ & & & & & & C4(6) & $C 4(6)$ & C4(6) & C4(6) & C8(11) & C8(11) & - & - \\
\hline & $\begin{array}{l}\text { After } \\
12 \mathrm{~m}\end{array}$ & & & & & & C4(6) & C4(6) & C4(6) & C4(6) & C8(11) & C8(11) & - & - \\
\hline
\end{tabular}

\section{Cell isolation and transplantation}

Following daycare hospitalization, SCs and MSCs were extracted from the patients in the operating room under sterile conditions and they were discharged immediately after the procedure.

In order to collect SCs, as we previously reported (13), the sural nerve of the patient posterior to lateral malleolus was cut and sliced into 1- to 2- mm pieces, then, was incubated with collagenase (1.4 U ml - 1; Sigma, St. Louis, MO, USA) and Dispase (2.4 U ml - 1; Sigma, USA). After washing the collagenase for two times with DMEM/F12 and mesh filtering, the cells were treated with DMEM/F12, not including fetal bovine serum (FBS, Gibco, USA) for $5 \mathrm{~d}$ (37C, $5 \%$ CO2). Then, we increased the concentration of FBS in culture progressively up to $10 \%$ during the period of 1 week. The characterization of the isolated cells was approved by S-100 immunocytological staining, as described in our previous study (13).

In order to isolate bone marrow MSC, bone marrow blood $(100-150 \mathrm{~mL})$ was aspirated from the iliac bone. After the samples underwent a density gradient by Ficoll (1.077 g/L, Sigma, USA) at the ratio of 1:3, the mononuclear cell layer was recovered from the gradient interface after bone marrow blood was centrifuged (400 g for $40 \mathrm{~min}$ ). To separate the platelets and mononuclear cells, the cells were centrifuged three times with less gradient and time. We assessed the cell surface markers (CD73, CD105, CD45, and CD34) through flow cytometry analysis to ensure the characteristic of the isolated cells.

The MSCs and SCs were cultured separately and a mixture of the isolated cells was composed of MSCs at the final concentration of $5 \times 10^{5}$ cells per ml and SCs at the final concentration of $5 \times 10^{5}$ cells per $\mathrm{ml}$ (total volume of $6 \mathrm{ml}$ ) was prepared.

To transplant cells, the patients were daycare hospitalized (approximately 3 weeks after biopsy procedure). We transplanted the mixture of MSCs and SCs into the L4/L5 level in the operation room through lumbar puncture using spinal needle $24 \mathrm{G}$. We assured the entrance to the subarachnoid space by the existing CSF from the spinal needle. The mixture of cells $(6 \mathrm{ml})$ was slowly injected. We kept the needle in place for 1 min to avoid leakage. The patients were discharged $1 \mathrm{~h}$ after procedure.

\section{Follow-up procedure}

The patients were involved in 12-month follow-up process after cell injection. We assessed neurological changes by the ASIA sensory-motor scale, functional recovery by the spinal cord independence measure (SCIM-III), and subjective changes along with adverse events (AE) with our checklist, presented in Table 2. Furthermore, electromyography (EMG), nerve conduction velocity (NCV), magnetic resonance imaging (MRI), and uroynamic study (UDS) were conducted for all the patients at the baseline, 6 months, and one year after the intervention.

We also ensured that all the participants received standard therapy for $\mathrm{SCl}$ injuries such as regular rehabilitation programs.

\section{Statistical analysis}

To study the significance of changes in the clinical scales, Wilcoxon rank test was used in SPSS 16.0 (IBM Crop., Armonk, USA). When the results were deemed statistically significant, the size of the effect was calculated manually using the following formula

$$
\mathrm{r}=\frac{z}{\sqrt{N}} \text { (N= Observations) }
$$

\section{Result}

\section{Cell assessments}

The extracted cells had the same characteristics as the cells we used in our previous study (13). Cells isolated from the sural nerve were positive for S-100 marker, which indicated that these cells had the properties of SCs (Fig. 1.a). MSCs were positive for CD73 and CD 105 and negative for CD45 and CD34 in flow cytometry analysis (Fig. 1.b).

\section{Adverse events}

Several mild adverse events were observed among the patients. Increasing in spasticity, numbness, or tingling sensation and neuropathic pain was reported by 5,4 , and 2 out of 11 patients, respectively. Headache and facial flushing appeared in two patients after transplantation that was resolved spontaneously. Furthermore, none of the patients reported fever after injection (Table 2). 


\begin{tabular}{|c|c|c|c|c|c|c|c|c|c|c|c|c|c|c|c|c|c|c|}
\hline \multirow{2}{*}{\multicolumn{2}{|c|}{$\begin{array}{l}\text { Patient } \\
\text { number }\end{array}$}} & \multicolumn{7}{|c|}{ ADVERSE EVENT } & \multirow{3}{*}{$\begin{array}{c}\begin{array}{c}\text { Trunk } \\
\text { movements }\end{array} \\
-\end{array}$} & \multirow{3}{*}{$\begin{array}{c}\text { Equilibrium In sitting } \\
\text { position } 7 \\
--\end{array}$} & \multicolumn{7}{|c|}{ SUBJECTIVE CHANGES } & \multirow{3}{*}{$\begin{array}{c}\begin{array}{c}\text { Regularity of } \\
\text { bowel } \\
\text { movement }\end{array} \\
-\end{array}$} \\
\hline & & Fever & $\begin{array}{c}\text { Numbneas or } \\
\text { tinglling senaation }\end{array}$ & $\begin{array}{l}\text { Faclal } \\
\text { fluening }\end{array}$ & Headache & General ache & $\begin{array}{l}\text { Neuropathic } \\
\text { paln }\end{array}$ & spasticlty & & & $\begin{array}{l}\text { Equilibrium In standing c } \\
\text { position } 7\end{array}$ & Constipation & $\begin{array}{l}\text { Urination } \\
\text { eenastion }\end{array}$ & Volding & Consiatency & $\begin{array}{l}\text { Feelling of } \\
\text { defecation }\end{array}$ & $\begin{array}{c}\text { Bowel sphincter } \\
\text { management }\end{array}$ & \\
\hline \multirow{4}{*}{1} & Before & - & - & - & - & - & - & - & & & + & + & - & - & - & - & - & \\
\hline & $\begin{array}{c}\text { After } \\
8 \mathrm{~m}\end{array}$ & - & + & - & - & - & + & + & - & - & $\downarrow$ & $\downarrow$ & + & - & - & - & - & + \\
\hline & $\begin{array}{l}\text { After } \\
12 \mathrm{~m}\end{array}$ & - & + & - & - & - & - & $\uparrow$ & + & - & $\downarrow$ & $\downarrow$ & + & - & - & + & - & - \\
\hline & Before & - & - & - & - & - & - & - & + & + & + & + & - & - & + & - & - & + \\
\hline \multirow[t]{3}{*}{2} & After & - & - & - & - & + & + & + & $\uparrow$ & $\uparrow$ & + & + & - & - & + & - & - & + \\
\hline & $\begin{array}{l}\text { After } \\
12 \mathrm{~m}\end{array}$ & - & - & - & - & + & + & $\uparrow$ & $\uparrow$ & $\uparrow$ & + & + & - & - & + & - & - & + \\
\hline & Before & - & + & - & - & - & - & - & + & + & + & + & - & - & - & - & - & - \\
\hline \multirow[t]{3}{*}{3} & $\begin{array}{l}\text { After } \\
8 \mathrm{~m}\end{array}$ & - & $\uparrow$ & + & - & - & - & + & $\uparrow$ & $\uparrow$ & $\downarrow$ & $\downarrow$ & - & - & - & - & - & - \\
\hline & $\begin{array}{l}\text { After } \\
12 \mathrm{~m}\end{array}$ & - & $\uparrow$ & + & - & - & - & $\uparrow$ & $\uparrow$ & $\uparrow$ & $\downarrow$ & $\downarrow$ & - & - & - & - & - & - \\
\hline & Before & - & + & - & - & - & - & + & - & - & - & - & - & - & - & - & - & - \\
\hline \multirow[t]{3}{*}{4} & $\begin{array}{l}\text { After } \\
8 \mathrm{~m}\end{array}$ & - & + & - & - & - & - & + & - & - & - & - & - & - & - & - & - & - \\
\hline & $\begin{array}{l}\text { After } \\
12 \mathrm{~m}\end{array}$ & - & + & - & - & - & - & + & - & - & - & - & - & - & - & - & - & - \\
\hline & Before & - & - & - & - & - & + & - & + & + & + & + & - & - & - & + & + & - \\
\hline \multirow[t]{3}{*}{5} & $\begin{array}{l}\text { After } \\
8 \mathrm{~m}\end{array}$ & - & - & - & - & - & + & - & $\uparrow$ & + & + & + & - & + & - & + & + & - \\
\hline & $\begin{array}{l}\text { After } \\
12 \mathrm{~m}\end{array}$ & - & - & - & - & - & $\downarrow$ & - & $\uparrow$ & + & $\downarrow$ & $\downarrow$ & - & + & - & + & + & - \\
\hline & Before & - & - & - & - & - & - & - & + & - & - & - & - & - & - & - & - & + \\
\hline \multirow[t]{3}{*}{6} & $\begin{array}{l}\text { After } \\
8 \mathrm{~m}\end{array}$ & - & - & - & - & - & - & - & $\uparrow$ & + & - & - & - & - & - & - & - & + \\
\hline & $\begin{array}{l}\text { After } \\
12 \mathrm{~m}\end{array}$ & - & - & - & - & - & - & - & $\uparrow$ & $\uparrow$ & - & - & - & - & - & - & - & + \\
\hline & Before & - & - & + & - & - & + & + & + & - & - & - & - & - & - & - & - & - \\
\hline \multirow[t]{3}{*}{7} & $\begin{array}{l}\text { After } \\
8 \mathrm{~m}\end{array}$ & - & - & + & - & - & + & $\uparrow$ & $\uparrow$ & + & - & - & - & - & - & - & - & - \\
\hline & $\begin{array}{l}\text { After } \\
12 \mathrm{~m}\end{array}$ & - & - & + & - & - & + & $\uparrow$ & $\uparrow$ & $\uparrow$ & - & - & - & - & - & - & - & - \\
\hline & Before & - & - & + & - & - & - & + & + & - & + & + & - & - & - & - & - & - \\
\hline \multirow[t]{3}{*}{8} & $\begin{array}{l}\text { After } \\
8 \mathrm{~m}\end{array}$ & - & + & + & - & - & - & $\uparrow$ & $\uparrow$ & + & + & + & - & - & - & - & - & - \\
\hline & $\begin{array}{l}\text { After } \\
12 \mathrm{~m}\end{array}$ & - & + & + & - & - & - & $\uparrow$ & $\uparrow$ & $\uparrow$ & + & + & - & - & - & - & - & - \\
\hline & Before & - & - & - & - & - & - & + & - & - & - & - & - & - & - & - & - & - \\
\hline \multirow[t]{3}{*}{9} & $\begin{array}{l}\text { After } \\
8 \mathrm{~m}\end{array}$ & - & - & - & - & - & - & + & + & + & - & - & + & - & - & + & - & - \\
\hline & $\begin{array}{l}\text { After } \\
12 \mathrm{~m}\end{array}$ & - & - & - & - & - & - & + & $\uparrow$ & $\uparrow$ & - & - & + & - & - & + & - & - \\
\hline & Before & - & + & - & - & - & - & - & + & - & + & + & - & - & - & - & - & + \\
\hline \multirow{2}{*}{10} & $\begin{array}{l}\text { After } \\
8 \mathrm{~m}\end{array}$ & - & $\uparrow$ & - & - & - & - & - & + & + & + & + & - & - & - & - & - & + \\
\hline & Before & - & + & - & - & - & - & + & - & - & - & - & - & - & - & - & - & - \\
\hline \multirow{2}{*}{11} & $\begin{array}{l}\text { After } \\
8 \mathrm{~m}\end{array}$ & - & + & - & - & - & - & + & - & - & - & - & - & - & - & - & - & - \\
\hline & $\begin{array}{l}\text { After } \\
12 \mathrm{~m}\end{array}$ & - & + & - & - & - & - & + & - & - & - & - & - & - & - & - & - & - \\
\hline
\end{tabular}

Other systemic complications such as anaphylactic shock, hypersensitivities, rush, or inflammation were not observed. Infectious complications associated with transplantation-like meningitis were not evident in the study.

Although previous spinal instrumentation caused some worst effects on the visibility of images in the patients, MRI indicated no neoplastic overgrowth, syringomyelia, or pseudo-meningocele after transplantation (Fig. 2).

\section{ASIA evaluation}

Sensory and/or motor improvement was evident in 9 patients according to ASIA assessment. Six patients experienced positive sensory changes and four patients had motor recovery (Table 1). In our assessment, the cervical SCI patients showed more improvement rate in motor aspects (75\% of the cervical and $14 \%$ of the lumbar-thoracic patients had motor improvement) and lumbar-thoracic SCI patients experienced more improvement rate in sensory ASIA score (63\% of the lumbar-thoracic and $50 \%$ of the cervical patients had sensory improvement) (Fig. 3 ).

In terms of the intensity of the changes, light touch ASIA score alterations were approximately the same as the pinprick changes ( $11.6 \pm 13.1$ and $12 \pm 13$,

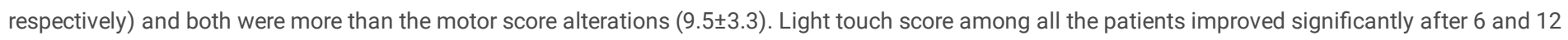
months in comparison with pre-transplantation scores ( $p$-value $=0.042$ and 0.027 , respectively). Score differences between $6^{\text {th }}$ and $12^{\text {th }}$ months were not significant $(p$-value $=0.317)$. For pinprick, the results were the same as the light touch changes $(p$-value $=0.041,0.027$, and 0.317 , respectively). The difference between the motor score in pre-transplantation evaluation and 6- and 12-months follow up was not significant ( $p$-value $=0.068$ and 0.066 , respectively) (Fig. 3, Table 3). 


\begin{tabular}{|c|c|c|c|c|c|}
\hline & Score Subject & Population & Time Mean & $\mathrm{SD}$ & P-value \\
\hline \multirow[t]{27}{*}{ SIA } & \multirow[t]{9}{*}{ Motor Score } & \multirow[t]{3}{*}{ Total Study Population } & a Before Injection 37.54 & 17.58 & - \\
\hline & & & After 6 months 40.45 & 514.80 & 0.068 \\
\hline & & & After 12 months 41.00 & 14.58 & 0.066 \\
\hline & & \multirow[t]{3}{*}{ Thoracic Patients } & Before Injection 50.00 & 00.00 & - \\
\hline & & & After 6 months 51.00 & 2.64 & 0.317 \\
\hline & & & After 12 months 51.28 & 3.40 & 0.317 \\
\hline & & \multirow[t]{3}{*}{ Cervical Patients } & Before Injection 15.75 & 55.90 & - \\
\hline & & & After 6 months 22.00 & 1.63 & 0.109 \\
\hline & & & After 12 months 23.00 & 2.58 & 0.109 \\
\hline & \multirow[t]{9}{*}{ Light Touch } & \multicolumn{3}{|c|}{ Total Study Population Before Injection 47.6324 .37} & - \\
\hline & & \multirow{5}{*}{ Thoracic Patients } & After 6 months 50.54 & 24.46 & $0.042^{*}$ \\
\hline & & & After 12 months 51.80 & 25.74 & $0.027^{*}$ \\
\hline & & & Before Injection 60.57 & 719.51 & - \\
\hline & & & After 6 months 63.71 & 17.12 & 0.109 \\
\hline & & & After 12 months 68.00 & 16.44 & 0.068 \\
\hline & & \multirow[t]{3}{*}{ Cervical Patients } & Before Injection 25.00 & 12.05 & - \\
\hline & & & After 6 months 27.50 & 15.00 & 0.180 \\
\hline & & & After 12 months 27.50 & 15.00 & 0.180 \\
\hline & \multirow[t]{9}{*}{ Pin Prick } & \multirow[t]{3}{*}{ Total Study Populatio } & Before Injection 48.54 & 25.66 & - \\
\hline & & & After 6 months 51.63 & 325.71 & $0.041 *$ \\
\hline & & & After 12 months 53.00 & 27.00 & $0.027^{*}$ \\
\hline & & \multirow[t]{3}{*}{ Thoracic Patients } & Before Injection 62.00 & 20.81 & - \\
\hline & & & After 6 months 65.42 & 219.13 & 0.102 \\
\hline & & & After 12 months 70.00 & 17.15 & 0.066 \\
\hline & & \multirow[t]{3}{*}{ Cervical Patients } & Before Injection 25.00 & 12.09 & - \\
\hline & & & After 6 months 27.50 & 15.86 & 0.180 \\
\hline & & & After 12 months 27.50 & 15.86 & 0.180 \\
\hline \multirow[t]{36}{*}{ M III } & Total Score & Total Study Populatio & Before Injection 28.9 & 12.99 & - \\
\hline & & & After 6 months 37.54 & 18.40 & $0.012 *$ \\
\hline & & & After 12 months 43.10 & 25.77 & $0.018^{*}$ \\
\hline & & Thoracic Patients & Before Injection 37.14 & 46.28 & - \\
\hline & & & After 6 months 49.28 & 37.08 & $0.018 *$ \\
\hline & & & After 12 months 60.50 & 14.18 & $0.028 *$ \\
\hline & & Cervical Patients & Before Injection 14.50 & 7.00 & - \\
\hline & & & After 6 months 17.00 & 12.00 & 0.317 \\
\hline & & & After 12 months 17.00 & 12.00 & 0.317 \\
\hline & Self-Care & Total Study Populatio & Before Injection 8.00 & 5.53 & - \\
\hline & & & After 6 months 10.36 & 6.74 & $0.043^{*}$ \\
\hline & & & After 12 months 11.40 & 7.93 & $0.005^{* *}$ \\
\hline & & Thoracic Patients & Before Injection 11.71 & 12.49 & - \\
\hline & & & After 6 months 14.71 & 2.98 & 0.068 \\
\hline & & & After 12 months 17.16 & 2.48 & $0.042 *$ \\
\hline & & Cervical Patients & Before Injection 1.50 & 1.00 & - \\
\hline & & & After 6 months 2.75 & 3.50 & 0.317 \\
\hline & & & After 12 months 2.75 & 3.50 & 0.317 \\
\hline & Respiration and Sphincter & tTotal Study Populatio & Before Injection 17.18 & 5.89 & - \\
\hline & & & After 6 months 21.72 & 28.22 & $0.016^{*}$ \\
\hline & & & After 12 months 26.20 & 13.63 & $0.027^{*}$ \\
\hline & & Thoracic Patients & Before Injection 19.57 & 74.64 & - \\
\hline & & & After 6 months 26.00 & 4.24 & $0.026^{*}$ \\
\hline & & & After 12 months 34.16 & 10.04 & $0.043^{*}$ \\
\hline & & Cervical Patients & Before Injection 13.00 & 6.00 & - \\
\hline & & & After 6 months 14.25 & 58.50 & 0.317 \\
\hline & & & After 12 months 14.25 & 58.50 & 0.317 \\
\hline & Mobility & Total Study Populatio & Before Injection 3.72 & 3.58 & - \\
\hline & & & After 6 months 5.45 & 4.78 & $0.039^{*}$ \\
\hline & & & After 12 months 8.90 & 12.81 & $0.043^{*}$ \\
\hline & & Thoracic Patients & Before Injection 5.85 & 2.60 & - \\
\hline & & & After 6 months 8.57 & 2.63 & $0.039 *$ \\
\hline & & & After 12 months 14.83 & 13.77 & $0.043^{*}$ \\
\hline & & Cervical Patients & Before Injection 0.00 & 0.00 & - \\
\hline & & & After 6 months 0.00 & 0.00 & 1.00 \\
\hline & & & After 12 months 0.00 & 0.00 & 1.00 \\
\hline
\end{tabular}

\section{SCIM III changes}

Regarding our SCIM III assessment, 8 out of 11 patients had some degrees of functional recovery, most of which were thoracic SCI patients. So, all the thoracic SCI patients experienced a positive change in SCIM III evaluation (Table 1). Among the cervical SCI patients, only one patient (number 8 ) had improvement in the "sphincter management-bladder" item. Mean \pm SD of the SCIM III changes was $21.2 \pm 13.3$ and, among the SCIM III sub-scores, "respiration

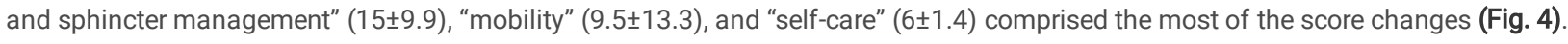


Statistical analysis revealed that the patients experienced significant progressive changes after each 6 months in SCIM III total score and its sub-scales (Fig. 4). Differences between the pre- and post-transplantation groups and between each post-transplantation group (cervical and thoracolumbar) were significant in total SCIM III score, respiration and sphincter management, mobility, and self-care (p-value of changes after 6 months were $0.012,0.043,0.016$, and 0.039 and, for changes after 12 months, were $0.018,0.005,0.027$, and 0.043 , respectively). In thoracolumbar patients, our statistical evaluation revealed that the differences between pre-transplantation and 6 and 12 months were significant (except self-care changes after 6 months, which was not significant). P-value for changes of SCIM III score in thoracolumbar SCI patients after 6 and 12 months were 0.018 and 0.028 , respectively (Table 3).

\section{Subjective outcomes}

Our findings supported that the most remarkable positive, subjective improvement was in the trunk movement (in 8 patients) and equilibrium in standing/sitting positions (in 7 patients). Furthermore, three patients (patients with numbers 1,3, and 5) experienced a reduction in the severity of constipation. We also observed that the two of our patients (numbers 1 and 9 ) claimed that they obtained the sensation of the filling bladder and rectum in $6^{\text {th }}$ and $12^{\text {th }}$ months of their follow-up (patient number 1 acquired sense of rectal filling in $12^{\text {th }}$ months of follow-up). Furthermore, one patient had successful changes as the empowerment of voiding (patient number 5) (Table 2).

UDS assessment of patient number 5 also supported this change. Pre-transplantation UDS of the patient revealed the presence of uninhibited contraction (no voiding), maximum detrusor pressure of $55 \mathrm{cmH} 20$, maximum flow during cytometry less than $1 \mathrm{cc} / \mathrm{sec}$, and post-voiding residue of $314 \mathrm{cc}$. Posttransplantation UDS changed to maximum detrusor pressure of $15 \mathrm{cmH} 2 \mathrm{O}$, maximum flow during cytometry of $3.3 \mathrm{cc} / \mathrm{sec}$, post-voiding residue of $410 \mathrm{cc}$, and voided volume of $40 \mathrm{cc}$.

\section{Discussion}

The present study showed that autologous SC and bone marrow-derived MSC transplantation at the subacute stage of SCl could reveal significant improvement in sensory and neurological function among the patients. Also, 75\% of the cervical patients showed some degrees of motor improvements and $50 \%$ of them had sensory changes. But, thoracolumbar SCI patients experienced more improvement rate in sensory ASIA score (63\% of the patients) and less in motor score (14\%). Two patients obtained the sensation of the bladder and rectal filling and one patient claimed that he acquired the ability of voluntary voiding. There were no systemic nor serious complications such as fever, anaphylactic shock, hypersensitivities, rush, or inflammation after autologous transplantation. Also, radiological images showed no neoplastic overgrowth, syringomyelia, or psuedomeningocele, which documents the safety of using this cell combination therapy at the subacute stage.

Current clinical trial used the combination of autologous SC and bone marrow-derived MSC transplantation because of multi-faceted inhibitory nature of the CNS lesion (19). After spinal cord injury, multiple changes occur in damaged tissues that require various treatment strategies like neuroprotection, axonal regeneration promotion, and rehabilitation (20).

Since one of the issues following spinal cord injury is an inflammatory environment, previous treatment strategies have focused on reducing inflammatory responses. In past, only high-dose methylprednisolone has been shown as the standard of care and has limited effectiveness for regenerating the spinal cord (21).

Cell transplantation is a targeted new promising therapeutic strategy for spinal cord regeneration based on a series of animal and clinical studies and it has been previously reported that stem cells have a potential effect for the SCI treatment (22-24). The safety and clinical application of SCs and MSCs separately have been reported in treatment of SCI patients $(7,8,25,26)$. MSCs are an appropriate source for cell therapy due to their ability of high growth rate, low immunogenicity, and a favorable ethical profile (27). Also, MSCs could enhance and support neurite outgrowth, axonal surviving, and remyelination (28). In spite of the beneficial effects of MSC transplantation, according to the previous findings, their remyelinating ability is inadequate in SCI patients (13). So far, some studies have performed combination therapies for spinal cord regeneration; using two types of effective cells (SCs and MSCs) for spinal cord regeneration is one of these combination methods $(20,29,30)$. In our previous work, we reported the safety of SCs and MSCs combination therapy in damaged chronic human spinal cord with no new deficit or adverse effect in the patients during short- and longer-term assessments (13). Park et al. evaluated the therapeutic effects of autologous bone marrow cell transplantation in conjunction with administering granulocyte macrophage-colony stimulating factor (GM-CSF) in six complete SCl patients and reported neurologic improvements in AIS grades (from A to C) in five patients (31).

The delivery time of the stem cells after spinal cord injury is important for better prognosis. This is because oligodendrocyte death and myelin loss after SCI are not a static phenomenon and demyelination continues and increases up to at least 450 days post-injury (34). Generally, 12-18 months post-injury optimally correspond with the natural history of neuro recovery; this time is the golden time for supporting and improving neuro recovery and promoting conditions for the long-term maintenance of health and function (18). In spite of promising results in chronic SCl patients after cell therapy (13, 35, 36), it seems that the sub-acute phase of stem cell transplantation after SCI may lead to better results (32). The study by Yoon et al. reported improvement in AIS grade of $30.4 \%$ of the acute and subacute treated patients (AIS A to B or C), whereas no significant changes were observed in the chronic patients. The majority of their study population had cervical injuries; nevertheless, they did not report the changes separately regarding the cervical and thoracic SCI patients (17). Some differences between our results and theirs could be explained by the different composition levels of injury and cell combination therapy in our study (33).

Among the 11 patients, one reported improvement in urination management after cell therapy. Likely, Jiang et al.'s study (10) showed 80\% of patients experienced recovery of urinary function. Nevertheless, Kishk et al. reported that no one in the treated or control groups reached complete recovery of bladder control (24). This controversy in the results could arise from different evaluation systems in each study. In spite of the importance of urinary problems in SCl, there was lack of comprehensive study for assessing cell transplantation in human subjects. 
Neuropathic pain is one of the irritable complications that increases after cell transplantation, so is observed in some patients based on our study and other previous works $(24,34)$. One of the possible reasons for neuropathic pain after cell therapy may be due to MSCs differentiation into astrocytes which could lead to increased CGRP-positive sprouting and a consequent hypersensitive state $(35,36)$. Because of small sample size and lack of control group, we could not conclude that the intrathecal injection of this combination cell therapy may increase neuropathic pain. So, clinical trials with larger volume sizes and control group are needed for definitive comment on this topic.

In the present study, we used SCs and MSCs combination therapy in complete subacute SCI subjects to assess the safety and efficacy of this intervention. Unlike our previous findings (13), the results of this treatment in subacute patients were promising for the patients' rehabilitation. Our data were in reasonably close agreement with the hopeful effects of combination cell therapy in sensory, motor, and functional status of subacute SCl patients alongside the positive subjective changes like empowerment in trunk movement, equilibrium in standing/sitting position, and sensation of the bladder and rectal filling. According to our limitations, we suggest a clinical trial with a large number of patients to unveil the effects of this kind of therapy on the suitable subgroups of SCI patients.

\section{Conclusion}

It seems that the combination of autologous SC and bone marrow-derived MSC transplantation at the subacute stage of SCl could significantly improve sensory and neurological function among the patients. As well as, there were no systemic nor serious complications after autologous transplantation. Large group studies are required to report the safety and efficacy of this combination.

\section{Declarations}

\section{Statement of Ethics}

The present study was conducted with the approval of the Ethics in Medical Research Committee of the Shahid Beheshti University of Medical Sciences (code of ethics: 106, registered October 2, 2018, ethics.research.ac.ir). All procedures were performed after written informed consent was obtained from all patients.

\section{Consent for publication}

Consent for publication was obtained from all patients.

\section{Acknowledgment}

None to declare.

\section{Conflicts of Interest}

The authors have no conflicts of interest to declare.

\section{Funding}

None to declare.

\section{Data Sharing}

Researchers could submit their research proposals to the corresponding author to access to datasets of this clinical trial.

\section{Author Contribution}

MHAP, SOY, MG, and MOY prepared the original draft. MHAP and MH conducted the formal analysis on data. SOY, MG and MSZ reviewed and finalized all drafts. MHAP, SOY, and FA conceptualized of the study and SOY, MEA and ARZ provided overall supervision. MRS and MEA all authors were involved in investigation and provided guidance on methodology. Also they reviewed and revised the manuscript and approved the final revision of the manuscript.

\section{References}

1. Furlan JC, Noonan V, Singh A, Fehlings MG. Assessment of impairment in patients with acute traumatic spinal cord injury: a systematic review of the literature. Journal of neurotrauma. 2011;28(8):1445-77.

2. James SL, Theadom A, Ellenbogen RG, Bannick MS, Montjoy-Venning W, Lucchesi LR, et al. Global, regional, and national burden of traumatic brain injury and spinal cord injury, 1990-2016: a systematic analysis for the Global Burden of Disease Study 2016. The Lancet Neurology. 2019;18(1):56-87.

3. Gurcay E, Bal A, Eksioglu E, Cakci A. Quality of life in patients with spinal cord injury. International journal of rehabilitation research Internationale Zeitschrift fur Rehabilitationsforschung Revue internationale de recherches de readaptation. 2010;33(4):356-8.

4. Fehlings MG, Vawda R. Cellular treatments for spinal cord injury: the time is right for clinical trials. Neurotherapeutics. 2011;8(4):704-20.

5. Jin MC, Medress ZA, Azad TD, Doulames VM, Veeravagu A. Stem cell therapies for acute spinal cord injury in humans: a review. Neurosurgical focus. 2019;46(3):E10.

6. Gazdic M, Volarevic V, Harrell C, Fellabaum C, Jovicic N, Arsenijevic N, et al. Stem cells therapy for spinal cord injury. International journal of molecular sciences. 2018;19(4):1039. 
7. Saberi H, Moshayedi P, Aghayan H-R, Arjmand B, Hosseini S-K, Emami-Razavi S-H, et al. Treatment of chronic thoracic spinal cord injury patients with autologous Schwann cell transplantation: an interim report on safety considerations and possible outcomes. Neuroscience letters. 2008;443(1):46-50.

8. Yoon SH, Shim YS, Park YH, Chung JK, Nam JH, Kim MO, et al. Complete spinal cord injury treatment using autologous bone marrow cell transplantation and bone marrow stimulation with granulocyte macrophage-colony stimulating factor: phase I/II clinical trial. Stem cells. 2007;25(8):2066-73.

9. Krause D. Plasticity of marrow-derived stem cells. Gene therapy. 2002;9(11):754-8.

10. Jiang P-C, Xiong W-P, Wang G, Ma C, Yao W-Q, Kendell SF, et al. A clinical trial report of autologous bone marrow-derived mesenchymal stem cell transplantation in patients with spinal cord injury. Experimental and therapeutic medicine. 2013;6(1):140-6.

11. Cofano F, Boido M, Monticelli M, Zenga F, Ducati A, Vercelli A, et al. Mesenchymal Stem Cells for Spinal Cord Injury: Current Options Limitations, and Future of Cell Therapy. International journal of molecular sciences. 2019;20(11):2698.

12. Suzuki Y, Ishikawa N, Omae K, Hirai T, Ohnishi K, Nakano N, et al. Bone marrow-derived mononuclear cell transplantation in spinal cord injury patients by lumbar puncture. Restorative Neurology and Neuroscience. 2014;32(4):473-82.

13. Yazdani SO, Hafizi M, Zali A-R, Atashi A, Ashrafi F, Seddighi A-S, et al. Safety and possible outcome assessment of autologous Schwann cell and bone marrow mesenchymal stromal cell co-transplantation for treatment of patients with chronic spinal cord injury. Cytotherapy. 2013;15(7):782-91.

14. Franklin RJ, Gilson JM, Blakemore WF. Local recruitment of remyelinating cells in the repair of demyelination in the central nervous system. Journal of neuroscience research. 1997;50(2):337-44.

15. Oudega M, Xu X-M. Schwann cell transplantation for repair of the adult spinal cord. Journal of neurotrauma. 2006;23(3-4):453-67.

16. Kanno H, Pressman Y, Moody A, Berg R, Muir EM, Rogers JH, et al. Combination of engineered Schwann cell grafts to secrete neurotrophin and chondroitinase promotes axonal regeneration and locomotion after spinal cord injury. Journal of Neuroscience. 2014;34(5):1838-55.

17. Yoon SH, Shim YS, Park YH, Chung JK, Nam JH, Kim MO, et al. Complete spinal cord injury treatment using autologous bone marrow cell transplantation and bone marrow stimulation with granulocyte macrophage-colony stimulating factor: Phase I/II clinical trial. Stem Cells. 2007;25(8):2066-73.

18. Burns AS, Marino RJ, Kalsi-Ryan S, Middleton JW, Tetreault LA, Dettori JR, et al. Type and timing of rehabilitation following acute and subacute spinal cord injury: a systematic review. Global spine journal. 2017;7(3_suppl):175S-94S.

19. McCreedy DA, Sakiyama-Elbert SE. Combination therapies in the CNS: engineering the environment. Neuroscience letters. 2012;519(2):115-21.

20. Bunge MB. Novel combination strategies to repair the injured mammalian spinal cord. The journal of spinal cord medicine. 2008;31(3):262-9.

21. Bracken MB, Holford TR. Effects of timing of methylprednisolone or naloxone administration on recovery of segmental and long-tract neurological function in NASCIS 2. Journal of neurosurgery. 1993;79(4):500-7.

22. Geffner L, Santacruz P, Izurieta M, Flor L, Maldonado B, Auad A, et al. Administration of autologous bone marrow stem cells into spinal cord injury patients via multiple routes is safe and improves their quality of life: comprehensive case studies. Cell transplantation. 2008;17(12):1277-93.

23. Nishida H, Nakayama M, Tanaka H, Kitamura M, Hatoya S, Sugiura K, et al. Evaluation of transplantation of autologous bone marrow stromal cells into the cerebrospinal fluid for treatment of chronic spinal cord injury in dogs. American journal of veterinary research. 2011;72(8):1118-23.

24. Kishk NA, Gabr H, Hamdy S, Afifi L, Abokresha N, Mahmoud H, et al. Case control series of intrathecal autologous bone marrow mesenchymal stem cell therapy for chronic spinal cord injury. Neurorehabilitation and neural repair. 2010;24(8):702-8.

25. Karamouzian S, Nematollahi-Mahani SN, Nakhaee N, Eskandary H. Clinical safety and primary efficacy of bone marrow mesenchymal cell transplantation in subacute spinal cord injured patients. Clinical neurology and neurosurgery. 2012;114(7):935-9.

26. Bhanot Y, Rao S, Ghosh D, Balaraju S, KV SK. Autologous mesenchymal stem cells in chronic spinal cord injury. British journal of neurosurgery. 2011;25(4):516-22.

27. Yorukoglu AC, Kiter A, Akkaya S, Satiroglu-Tufan NL, Tufan AC. A concise review on the use of mesenchymal stem cells in cell sheet-based tissue engineering with special emphasis on bone tissue regeneration. Stem cells international. 2017;2017.

28. Shende P, Subedi M. Pathophysiology, mechanisms and applications of mesenchymal stem cells for the treatment of spinal cord injury. Biomedicine \& Pharmacotherapy. 2017;91:693-706.

29. Wilems TS, Pardieck J, lyer N, Sakiyama-Elbert SE. Combination therapy of stem cell derived neural progenitors and drug delivery of anti-inhibitory molecules for spinal cord injury. Acta biomaterialia. 2015;28:23-32.

30. Rosner J, Avalos P, Acosta F, Liu J, Drazin D. The potential for cellular therapy combined with growth factors in spinal cord injury. Stem Cells International. 2012;2012.

31. Park HC, Shim YS, Ha Y, Yoon SH, Park SR, Choi BH, et al. Treatment of complete spinal cord injury patients by autologous bone marrow cell transplantation and administration of granulocyte-macrophage colony stimulating factor. Tissue engineering. 2005;11(5-6):913-22.

32. Hosseini SM, Sani M, Haider KH, Dorvash M, Ziaee SM, Karimi A, et al. Concomitant use of mesenchymal stem cells and neural stem cells for treatment of spinal cord injury: A combo cell therapy approach. Neuroscience letters. 2018;668:138-46.

33. Dai G, Liu X, Zhang Z, Yang Z, Dai Y, Xu R. Transplantation of autologous bone marrow mesenchymal stem cells in the treatment of complete and chronic cervical spinal cord injury. Brain research. 2013;1533:73-9.

34. Hofstetter CP, Holmström NA, Lilja JA, Schweinhardt P, Hao J, Spenger C, et al. Allodynia limits the usefulness of intraspinal neural stem cell grafts; directed differentiation improves outcome. Nature neuroscience. 2005;8(3):346-53.

35. Macias MY, Syring MB, Pizzi MA, Crowe MJ, Alexanian AR, Kurpad SN. Pain with no gain: allodynia following neural stem cell transplantation in spinal cord injury. Experimental neurology. 2006;201(2):335-48. 
36. Oraee-Yazdani S, Hafizi M, Atashi A, Ashrafi F, Seddighi A, Hashemi S, et al. Co-transplantation of autologous bone marrow mesenchymal stem cells and Schwann cells through cerebral spinal fluid for the treatment of patients with chronic spinal cord injury: safety and possible outcome. Spinal Cord. 2016;54(2):102-9.

\section{Figures}
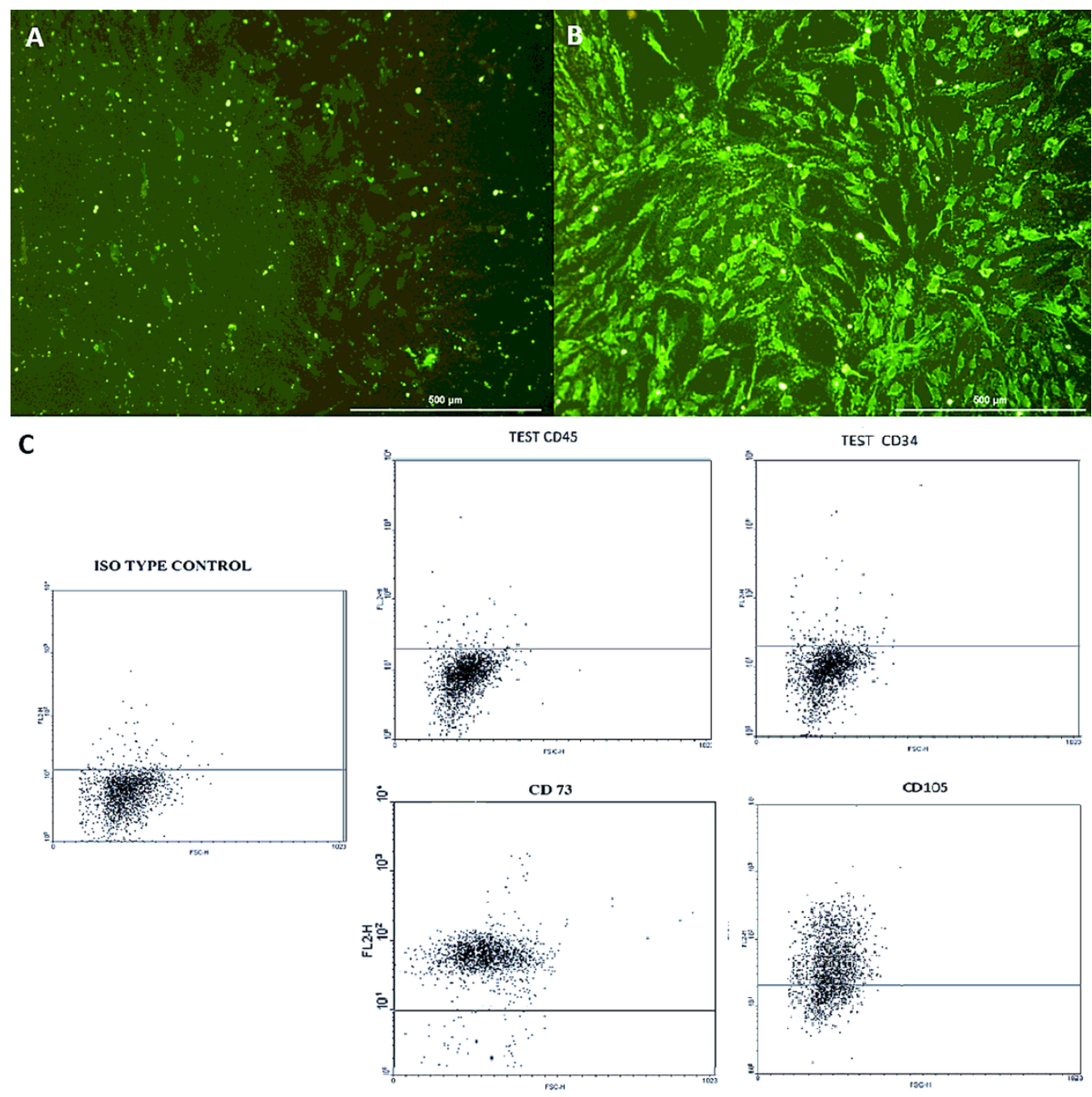

\section{Figure 1}

Results of bone marrow MSCs and SCs labratory assessments: A: S-100 immunocytological staining negative control; B: S-100 immunocytological staining test; C: The cell surface markers (CD73, CD105, CD45, and CD34) analysis through flow cytometry.

\section{Figure 2 placeholder}

\section{Figure 2}

Figure 2 was not provided with this version of the manuscript 


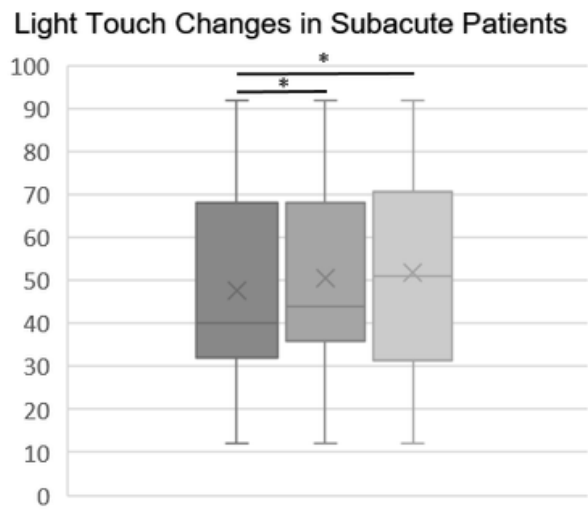

Light Touch Changes in Cervical Injuries
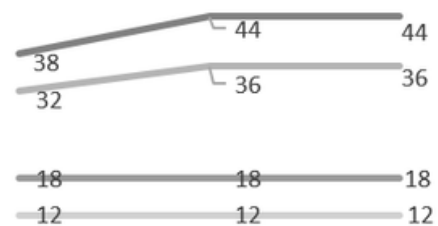

Pre After 6 months After 12 months
Pin Prick Changes in Subacute Patients

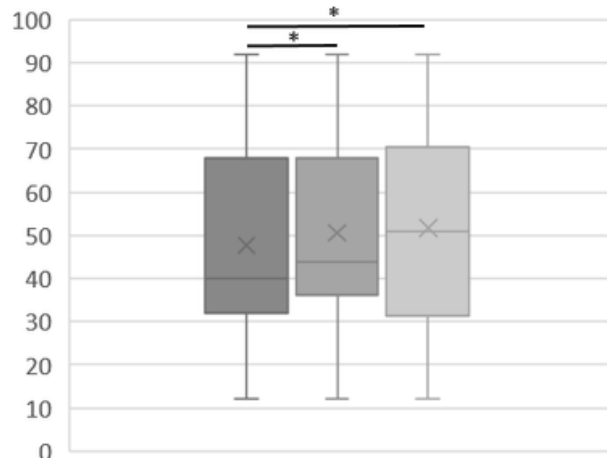

Pre $\square$ After 6 months $\square$ After 12 months
Motor Changes in Subacute Patients

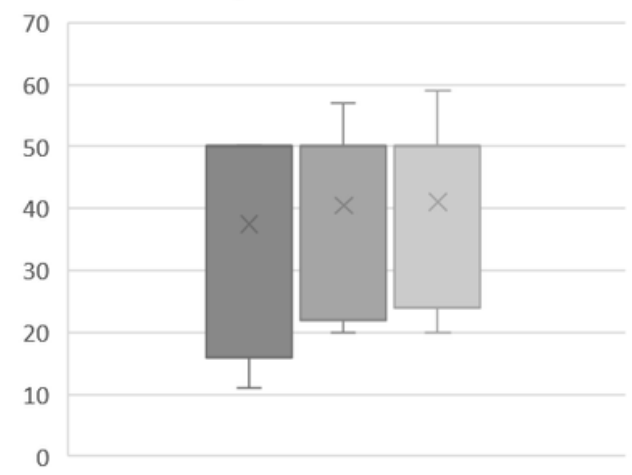

Pin Prick Changes in Cervical Injuries

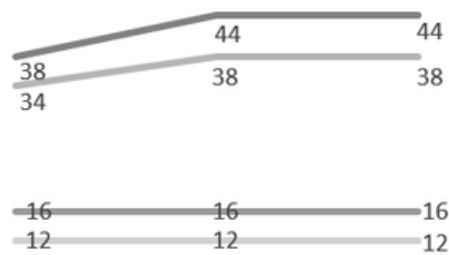

Pre After 6 months After 12 months

Patient 1

Patient 8
Motor Changes in Cervical Injuries

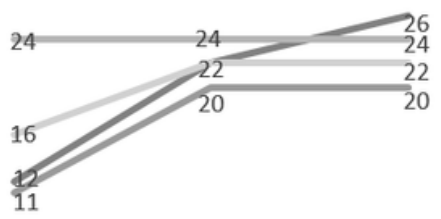

Pre
After 6 months After 12 months

\section{Figure 3}

Pre- and post-injection MRIs of patients with numbers 1,6, and 8: A: Patient number 1, pre-injection; B: Patient number 1, 6-month post-injection; C: Patient number 6, pre-injection; D: Patient number 6, 6-month post-injection; E: Patient number 8, pre-injection; F: Patient number 8, 6-month post-injection.
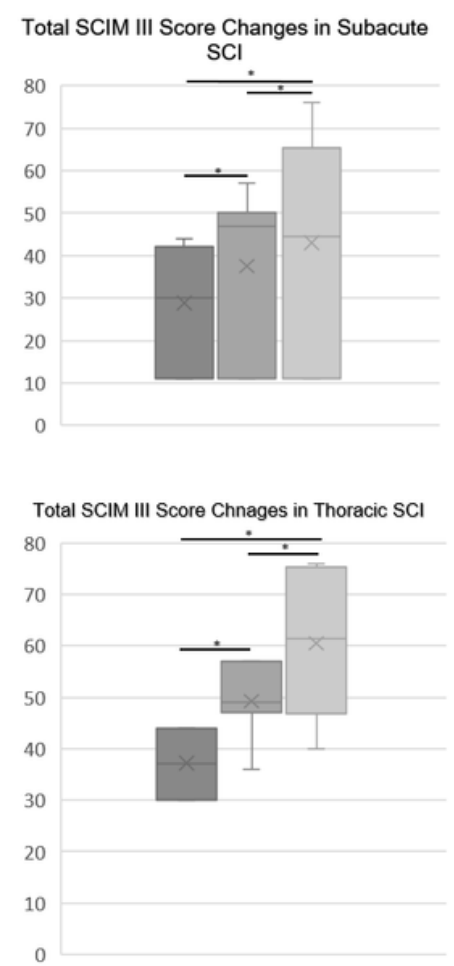
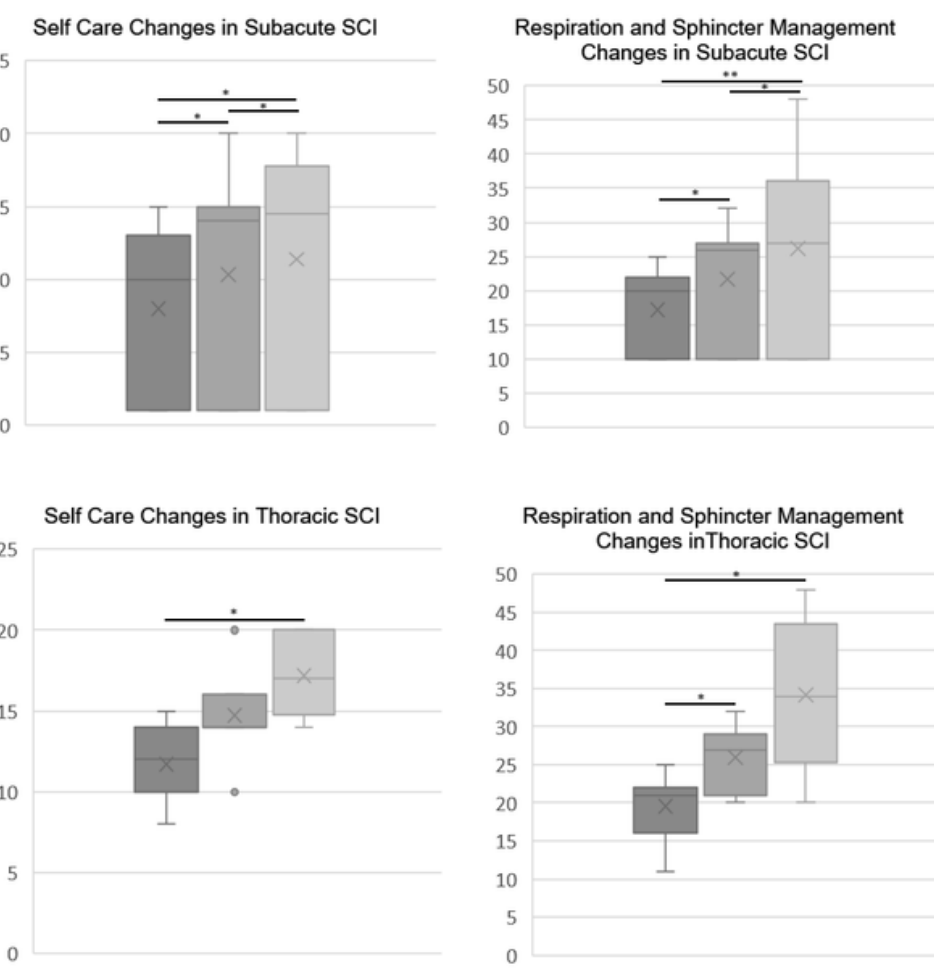

Respiration and Sphincter Management Changes inThoracic SCl

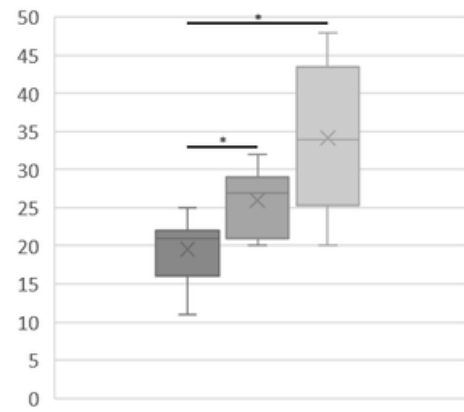

Mobility Changes in Subacute SCI

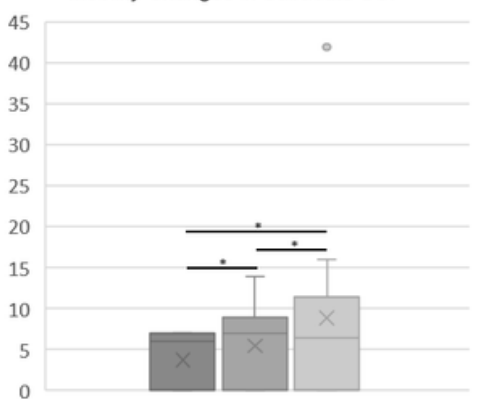

Mobility Changes in Thoracic SCl

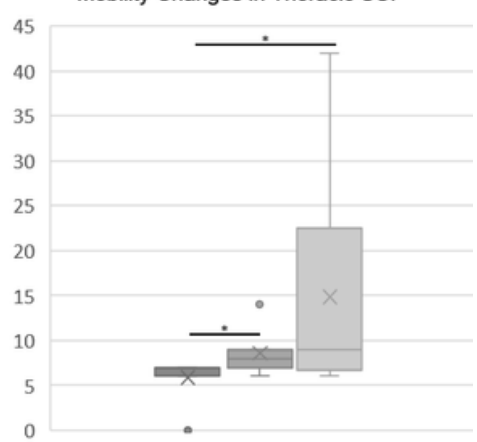


Figure 4

Total SCIMII score, self-care, respiration and sphincter management, and mobility changes in subacute and thoracic SCI patients. ( value $\leq 0.01)$ 